\title{
On the normalization of transport from ITG Modes
}

\author{
J. Weiland ${ }^{1}$ and A. Zagorodny ${ }^{2}$ \\ ${ }^{1}$ Chalmers University of Technology and EURATOM-VR Association, 41296 Gothenburg, Sweden \\ ${ }^{2}$ Bogoliubov Institute for Theoretical Physics, 03680 Kiev, Ukraine
}

(Received 24 August 2016; accepted 30 September 2016; published online 19 October 2016)

\begin{abstract}
We have analyzed the interplay of ITG turbulence and zonal flows as derived by the reductive perturbation method. Not surprisingly, the overall transport level depends strongly on the physics description of ITG modes. In particular, the fluid resonance turns out to play a dominant role for the excitation of zonal flows. This is the mechanism recently found to lead to the L-H transition and to the nonlinear Dimits upshift in transport code simulations. It is important that we have here taken the nonlinear temperature dynamics from the Reynolds stress as the convected diamagnetic flow. This has turned out to be the most relevant effect as found in transport simulations of the L-H transition, internal transport barriers, and Dimits shift. Published by AIP Publishing.

[http://dx.doi.org/10.1063/1.4964772]
\end{abstract}

\section{INTRODUCTION}

The problem of the normalization of drift wave turbulence has been a well known problem in turbulence simulations since it was discovered that drift wave turbulence, due to its quasi-2D nature, has an inverted cascade, ${ }^{1,2}$ feeding energy into the longest wavelengths as determined by the system size. The problem arises since, when the amplitude of the longest wavelengths becomes dominant, also the correlation length gets comparable with the system size thus leading to exceedingly strong transport. Thus, in the absence of a damping mechanism for long wavelengths, the transport may not saturate at all. Zonal flows ${ }^{3}$ or the similar more circular structure convection cells in drift wave turbulence have essentially two important effects. First, they are the natural mechanism for the damping of long wavelengths. Second, they are responsible for different types of transport barriers when active at the correlation length. In fact, experience from turbulence codes indicates that absorbing boundaries are needed for both long and short wavelengths in order to have the right experimental level of transport. The diffusivity will then be of the type

$$
\chi_{i}=\frac{\gamma^{3} / k_{r}{ }^{2}}{\omega_{r}{ }^{2}+\gamma^{2}} .
$$

Transport coefficients of the type (1) or its simplified Markovian version $\left(\gamma / \mathrm{k}_{\mathrm{r}}{ }^{2}\right)$ are the central parts of almost all transport coefficients discussed in the following. ${ }^{4-41}$ The type (1) was first obtained in Ref. 4 for the diagonal part of the thermal conductivity, with further details in Refs. 5-8 and later in Ref. 10. As it turns out, all we need to obtain the type (1) is to have a linear growth rate that is balanced by a nonlinear ExB convection which is entirely stabilizing. Thus, while the turbulence in Refs. 4-8 was of a reactive toroidal type, the turbulence in Refs. 9-11 was driven by collisions. Important aspects are also that the quasilinear derivations in Refs. 4 and 6-8 included also a Doppler shift due to the magnetic drift while the derivations in Refs. 5 and 10 were strongly nonlinear, the derivation in Ref. 10 confirming the validity of a quasilinear treatment here. The need for absorbing boundaries for both short and long wavelengths enters through the condition that ExB convection is entirely stabilizing.

Second, we know that transport barriers sometimes develop in tokamak plasma. Here, experience from transport codes, including the strongly nonlinear Reynolds stress, shows that zonal flows can trigger and maintain such barriers both at the edge ${ }^{34,36}$ (H-mode) and internally. ${ }^{35}$ In such cases, zonal flows have become important at the correlation length. The correlation length is determined by the mode number of largest linear growth rate as normalized by the drift frequency. This was found numerically already in Ref. 4 and was recently explained in Ref. 18. The same thing has recently been observed to be the reason for the nonlinear "Dimits" upshift, ${ }^{14}$ which is a nonlinear increase of the critical temperature gradient for transport. As recently found, it is the poloidal rotation driven by the temperature gradient that triggers both the internal transport barrier and the $\mathrm{L}-\mathrm{H}$ transition. ${ }^{34-36}$ However, here it is not the temperature gradient itself but rather the temperature length-scale $\mathrm{L}_{t}=-\mathrm{T} / \mathrm{dT} /$ $\mathrm{dr}$ which is important. This means that the triggering of internal and edge barriers is somewhat different. In our present model, zonal flows are enhanced by the fluid resonance in the energy equation. This has turned out to be important in particular, for the Dimits shift.

As we have seen, zonal flows play important roles for several types of transport barriers. An important aspect is here also that zonal flows are reducing the correlation length $^{26,27}$ which contributes to reducing transport. Because of this, zonal flows are also a leading candidate for providing the needed damping mechanism for waves with wavelength of the system size. Since zonal flows are driven by the ponderomotive force of the Reynolds stress, it is obvious that we need waves propagating in both poloidal directions in order to create nonlinear structures of the zonal flow type that do not vary in the poloidal direction. It was clearly demonstrated by Wakatani and Hasegawa ${ }^{11}$ that the turbulence of collisional drift waves does not saturate if we allow the 
propagation of drift waves in only one poloidal direction. It was also found that the saturation level of the turbulence increases if we disturb the symmetry between the two poloidal directions by including Landau damping. There are several different numerical codes giving different results for important phenomena. For the Dimits shift, however, we believe that a fully nonlinear kinetic model as used by Dimits $^{12}$ and our reactive fluid model ${ }^{14}$ are the only models which work. It is also very interesting to note that the Hasegawa-Wakatani model ${ }^{11}$ with zero ion temperature gives the same transport coefficient as the diagonal part of our transport coefficient ${ }^{4-8}$ for ion temperature, also omitting the Doppler shift due to the magnetic drift.

These models have the common feature of using a reactive fluid closure. For the Hasegawa-Wakatani model this is exact and comes as a consequence of zero ion temperature. In our case, it comes as a consequence of using only moments with sources in the experiment and treating this system self consistently. Clearly, adding a dissipative wave particle resonance in a fluid treatment means that we include infinitely many moments that are not treated self consistently. The reactive closure, with finite ion temperature, then leads to the same normalization of transport as in the Hasegawa-Wakatani system. ${ }^{10}$ In fact, the fluid resonance in the energy equation plays a central role in most phenomena of interest here. The fact that it should be treated as exact was pointed out in Ref. 17. Here we note that it leads to the same type of diffusivity as found in Ref. 10.

\section{A. Formulation}

We will in this work focus on transport in an advanced fluid model. ${ }^{4-8}$ This model has recently been able to reproduce the spin-up of poloidal momentum in internal transport barriers $^{35}$ and the L-H transition $^{36}$ in more complete versions. However, the basic trigger is, in both cases, the excitation of poloidal rotation by the Reynolds stress. The simplest case of this is the electrostatic case with Boltzmann electrons. This case is simple enough to be derived analytically. We will here recall the mechanism using the Reductive perturbation method. ${ }^{13,15,17,28}$

We start with a description of the equations used in simulations of the Dimits shift. The energy equation is

$$
\frac{3}{2} n_{j}\left(\frac{\partial}{\partial t}+\mathbf{v}_{\mathbf{j}} \cdot \nabla\right) \mathrm{T}_{\mathrm{j}}+\mathrm{P}_{\mathrm{j}} \nabla \cdot \mathbf{v}_{\mathbf{j}}=-\nabla \cdot \mathbf{q}_{j}
$$

with closure

$$
\mathbf{q}_{j}=\mathbf{q}_{* j}=\frac{5}{2} \frac{P_{j}}{m_{j} \Omega_{c j}}\left(\mathbf{e}_{\|} \times \nabla T_{j}\right) .
$$

Now using

$$
\nabla \cdot \mathbf{q}_{* j}=-\frac{5}{2} n \mathbf{v}_{* \mathbf{i}} \cdot \nabla T_{i}+\frac{5}{2} n \mathbf{v}_{\mathbf{D} i} \cdot \nabla T_{i}
$$

and taking into account cancellations between convective diamagnetic effects we obtain

$$
\frac{\delta T_{j}}{T_{j}}=\frac{\omega}{\omega-5 \omega_{D j} / 3}\left[\frac{2}{3} \frac{\delta n_{j}}{n}+\frac{\omega_{* e}}{\omega}\left(\eta_{j}-\frac{2}{3}\right) \frac{e \phi}{T_{e}}\right] .
$$

Eq. (2d) is fundamental for the model in general and in particular, for the spin-up of poloidal momentum through the Reynolds stress. The presence of the temperature dependence of the Reynolds stress has recently been recognized also by other authors. ${ }^{33}$ Eq. (2d) contains both convective and compressional effects in combination with the fluid resonance. If we apply these equations only for ions, taking Boltzmann electrons, we will get the toroidal ITG mode. We note that in this case, using quasi-neutrality in (2d) simplifies our equations very much and we get a second degree dispersion relation if we ignore parallel ion motion.

Thus Boltzmann electrons means

$$
\frac{\delta n_{e}}{T_{e}}=\widehat{\phi}=\frac{e \phi}{T_{e}} .
$$

We then get the quadratic local dispersion relation ${ }^{4,6}$

$$
\begin{gathered}
\frac{\omega_{* e}}{N_{i}}\left[\omega\left(1-\varepsilon_{n}\right)+\left(\eta_{i}-\frac{7}{3}+\frac{5}{3} \varepsilon_{n}\right) \omega_{D i}\right. \\
\left.-k^{2} \rho_{s}^{2}\left(\omega-\omega_{* i T}\right)\left(\frac{\omega}{\omega_{* e}}+\frac{5}{3 \tau} \varepsilon_{n}\right)\right]=1, \\
N_{i}=\omega^{2}-\frac{10}{3} \omega \omega_{D i}+\frac{5}{3} \omega_{D i}{ }^{2},
\end{gathered}
$$

where $\varepsilon_{\mathrm{n}}=2 \mathrm{~L}_{\mathrm{n}} / \mathrm{R}$. It is here straight forward to include parallel ion motion as ${ }^{6}$

$$
\mathrm{v}_{\| \mathrm{i}}=\frac{k_{\|}}{\omega m_{i}}\left(e \varphi+\delta T_{i}+T_{i} \frac{\delta n_{i}}{n}\right) .
$$

However, this leads to considerably more complicated equations.

This system has, with electron trapping, ${ }^{22}$ given transport in surprisingly good agreement with experiments applying standard quasilinear fluxes. ${ }^{22-24}$ Electron trapping also gives us particle and electron temperature transport, including possibilities for pinches. ${ }^{22,24}$ However, already the simple ITG mode had first given good agreement (except for Dimits shift) with nonlinear simulations in the Cyclone simulations. ${ }^{12}$ These results, of course, depend on the fluid closure which was recently verified in Ref. 21. Ref. 21 also resolves an apparent contradiction between Refs. 19 and 20 and 8 and 13. In Refs. 19 and 20 three slab ITG modes are considered while in Refs. 8 and 13 toroidal ITG modes are considered. While the gross oscillations between the modes are here due to three wave interaction, periodic waveparticle interaction effects are superimposed on these. This is shown in Refs. 6 and 7. The numerator of the kinetic integral is the same for slab and toroidal modes. However, for toroidal modes, the magnetic drift frequency is added in the denominator. This changes the phase of the wave-particle oscillations so that the wave particle resonance is destabilizing linearly for slab modes while it is stabilizing linearly for toroidal modes. 


\section{TRANSPORT AND BOUNDARY CONDITION FOR LONG WAVELENGTHS}

As mentioned above, we first applied standard quasilinear theory to the system (2)-(4). We then obtained the saturation level by balancing linear growth and nonlinear terms (we take $\omega=\omega_{\mathrm{r}}+\mathrm{i} \gamma$ )

$$
\gamma \delta T=\mathbf{v}_{E} \cdot \nabla \delta T .
$$

This gave for inverse correlation lengths

$$
\frac{e \varphi}{T_{e}}=\frac{\gamma}{\omega_{* e}} \frac{1}{k_{x} L_{n}} .
$$

We now generalize the usual wave breaking limit to include a finite eigenfrequency.

With the displacement

$$
\xi=-\frac{1}{i \omega} \mathbf{v}_{\mathbf{E}}=-\frac{1}{i \omega B}\left(\mathbf{e}_{\|} \times \nabla \phi\right),
$$

we find (cf. Ref. 6, p. 52)

$$
\left|\xi_{x}\right|=\frac{\gamma}{|\omega|} \frac{1}{k_{x}} .
$$

Here $\xi_{\mathrm{x}}$ acts as the step length in the transport. It is reduced by the real eigenfrequency. The usual mixing length expression for transport is then generalized to (cf. Ref. 6, p. 52)

$$
\chi_{i}=\frac{\gamma^{3} / k_{r}^{2}}{\omega_{r}^{2}+\gamma^{2}} .
$$

This result was directly confirmed numerically by nonlinear turbulence simulations ${ }^{4}$ but also later confirmed for particle transport using extensive analytic calculations and simulations. ${ }^{10}$ The common form (7) is due to having the corresponding balance at saturation (5) and extending a large eddy simulation (LES) up to the correlation length. It was also pointed out in Ref. 10 that the result is insensitive to the detailed structure of the high $\mathrm{k}$ spectrum. In (7) $\omega_{\mathrm{r}}$ is due to a rocking of the turbulent eddies which reduces transport. It was also found in Ref. 10 that the linear eigenfrequency here is a good approximation. This is a non-Markovian effect ${ }^{5}$ and (7) goes into the usual $\gamma / \mathrm{k}_{\mathrm{x}}{ }^{2}$ result by Kadomtsev when $\gamma^{2} \gg \omega_{\mathrm{r}}^{2}$.

We notice that the nonlinearity is entirely stabilizing in (5). This means that we have no reflections from the boundaries in $\mathrm{k}$-space. For long wavelengths, this requires zonal flows which are thus implicitly assumed in (6a).

The similarities between energy and continuity equations even lead us to derive a Hasegawa-Wakatani system for temperature dynamics as seen in Ref. 16. Of course the generation of transport barriers requires the turbulence to drive rotation. This is in our present model due to the Reynolds stress and this is a strongly nonlinear effect. Thus, the transport code is no longer quasilinear. The main trigger of transport barriers as well as of the Dimits shift is the spinup of the poloidal rotation as described by Eq. (8)

$$
\Gamma_{p}=\left\langle v_{E r} v_{\theta}\right\rangle=-D_{B}^{2} k_{r} k_{\theta} \frac{1}{2} \hat{\phi}^{*}\left[\hat{\phi}+\frac{1}{\tau} \hat{p}_{i}\right]+c . c .
$$

We here use only the ExB motion for convection while both ExB and diamagnetic drifts are convected. This leads to the presence of the pressure perturbation in (8) and in it the temperature perturbation is given by (2d). This gives us a feedback since an increased temperature gradient (increased flux) gives us an increased temperature perturbation which in turn increases the Reynolds stress, thus reducing the flux. When the flux is reduced, the temperature gradient is also reduced and ultimately the turbulent flux is turned off. This mechanism is the same for internal and edge transport barriers. The mechanisms for internal and edge transport barriers are, however, slightly different since it is actually the temperature length scale which is important for turning off the instability. At the edge there is no problem since the barrier is formed just inside the separatrix where the temperature is kept fixed. Thus a reduction in the temperature gradient (flux) directly gives a reduction of the length scale. For an internal barrier, however, the situation is different because there, in principle, the temperature and temperature gradient can decrease at the same time, thus keeping the length scale unchanged. However, if we have small magnetic shear at the barrier, the transport is small and the temperature is kept small by the fact that the transport increases outside this point due to increased magnetic shear. Another important point here is that the temperature perturbation (2d) contains the fluid resonance. This resonance is strongest for the reactive fluid resonance and will be reduced if we add a kinetic resonance as done in gyro-Landau fluid models. This has been directly shown by the Dimits upshift which is smaller in gyro-Landau fluid models. This is also the reason why gyro-Landau fluid models give too large transport, in general, a factor between 2 and 3 to large transport in the Cyclone tests. This has to be compensated by renormalization in transport codes using such models. ${ }^{29,30}$ This has been discussed in Progress in ITER Physics basis ${ }^{30}$ where this renormalization gave about a factor 2 increase in the fusion $\mathrm{Q}$ for pedestal temperature $4 \mathrm{KeV}$. We note that the mechanism used here was also the active trigger in the simulation of the L-H transition on EAST. ${ }^{36}$ There we obtained the same region for gradients on the H-mode pedestal as previously found by local, fully nonlinear turbulence simulations. ${ }^{31}$

The fact that it is zonal flows that give the damping of long wavelengths was particularly clear from the fully nonlinear turbulence simulations in Ref. 11. There it was found that no saturation of the turbulence (and accordingly transport) occurred if only modes propagating in the electron drift direction were included. However, when also modes propagating in the ion drift direction were included there was saturation. Obviously both directions are needed for creating modes of the zonal flow type with no poloidal variation due to nonlinear beating. It was also found that the transport increased when linear Landau damping was included. Again the interpretation that this introduced an asymmetry between the poloidal directions of propagation is obvious. In Ref. 10 there was an averaging over the poloidal variation leading to

$$
E(k)=\frac{1}{2} k \oint F(k) d \theta=\pi k\langle F(k)\rangle_{\theta} \equiv \frac{1}{2} L(k),
$$


where

$$
F(k)=\left\langle k^{2}\left|\varphi_{k}\right|^{2}\right\rangle
$$

and

$$
\mathbf{k}=k e^{i \theta}
$$

We introduce

$$
W(k)=A_{F} \int_{k_{0}}^{k} k^{2} L(k) d k+\frac{1}{2}\left[k^{2} D_{F}(k)\right]^{2},
$$

where $\mathrm{k}_{0}$ is the minimum $\mathrm{k}$ determined by the system size and $A_{F}$ is a constant of order 1 .

Analytical calculations in Ref. 10 lead to

$$
\frac{d}{d k} W(k)=\frac{2}{9 k}\left[\gamma_{L}(k)+\sqrt{\gamma_{L}(k)^{2}+6 W(k)}\right]^{2},
$$

where $\gamma_{\mathrm{L}}$ is the linear growth rate and the boundary condition

$$
W\left(k_{0}\right)=\frac{1}{2} \gamma_{L}\left(k_{0}\right)^{2}
$$

This analytical solution is consistent with (1) and is also in excellent agreement with numerical simulations in Ref. 10.

In the equations used in our transport code, (7) is just the kernel. Our full derivation uses the flux

$$
\Gamma_{r}=\left\langle\delta T_{i} \mathrm{v}_{\mathrm{Er}}\right\rangle=-\chi \frac{d T}{d r},
$$

where $\delta \mathrm{T}_{\mathrm{i}}$ is given by (2d) including all drift frequencies. As indicated above, flow shear leads to a damping of instabilities. This has been noticed early but the formula generally used today is the Waltz rule ${ }^{39,40}$

$$
\gamma \rightarrow \gamma-\omega_{E x B}
$$

where $\omega_{\mathrm{ExB}}$ is the flow shear rate proportional to the flow shear $\mathrm{d} \mathrm{V}_{\theta} / \mathrm{dr}$ in a cylindrical system. The simplest transport coefficient with Boltzmann electrons is then

$$
\chi_{i}=\frac{1}{\eta_{i}}\left(\eta_{i}-\frac{2}{3}-\frac{10}{9 \tau} \varepsilon_{n}\right) \frac{\left(\gamma-\omega_{E x B}\right)^{3} / k_{r}^{2}}{\left(\omega_{r}-\frac{5}{3} \omega_{D i}\right)^{2}+\left(\gamma-\omega_{E x B}\right)^{2}} .
$$

We notice that we recover (7) if we omit flow shear and off diagonal terms and finally ignore the Doppler shift due to the magnetic drift. The magnetic drift is due to the fact that the ions move with this drift velocity. The radial magnetic field is obtained from ion radial force balance

$$
\begin{gathered}
E_{r}=B_{\theta} \mathrm{V}_{\varphi}-B_{\varphi} \mathrm{V}_{\theta}+\frac{1}{e Z n_{i}} \frac{\partial P_{i}}{\partial r}, \\
\omega_{E \times B}=\frac{r}{q} \frac{\partial}{\partial r}\left(q V_{E \theta} / r\right),
\end{gathered}
$$

where $\mathrm{V}_{\mathrm{e} \theta}=-\mathrm{E}_{\mathrm{r}} / \mathrm{B}$. In our code we simulate both poloidal and toroidal fluid fluxes which are then inserted into (12) to give the radial electric field. Then the flow shear is calculated from (13). As it turns out, it is usually the poloidal rotation which triggers particularly interesting phenomena like internal and edge transport barriers and Dimits shift. We will thus focus on the poloidal flux, given by (8) where we add neoclassical damping. The transport equation for poloidal flux in our code is then

$$
\frac{\partial U_{\theta}}{\partial t}+\frac{\partial}{\partial r} \Gamma_{p}=S_{v}
$$

Here the source will contain neoclassical damping.

\section{ZONAL FLOWS}

Eq. (8) is the equation we use for numerical calculation of the poloidal rotation. It is also possible to make the calculation analytically using a suitable ordering scheme. For simplicity, we will here show this without including electron trapping. We then use the Reductive perturbation scheme ${ }^{28}$ in the nonresonant case

$$
\begin{aligned}
f= & \sum_{\alpha} \sum_{l} \varepsilon^{\alpha} f_{l}^{\alpha}(x, \xi, \varsigma) e^{i l\left[k_{\|} z+k_{y} y-\omega t\right]}+c . c \\
& +\sum_{n} \varepsilon^{2 n} f_{0}{ }^{4 / 3+(2 / 3) n}(x, \xi, \varsigma),
\end{aligned}
$$

where we used standard slab coordinates and $\mathrm{x}=\mathrm{x}$, $\xi=\varepsilon(\mathrm{y}-\lambda \mathrm{t})$, and $\zeta=\varepsilon^{2} \mathrm{t}$. In the present nonresonant ordering, $\zeta$ does not enter. In (15) $\mathrm{z}$ is the toroidal coordinate, which is ignorable in the linear calculations and which is multiplied by its complex conjugate to obtain the nonlinearly driven zonal flow. Thus, $\mathrm{z}$ does not appear explicitly in the following. Here $\varepsilon \ll 1$ is the expansion parameter and $\lambda$ is a convective velocity which will turn out to be the group velocity. We have here given the nonresonant ordering, as used in Ref. 15. However, its stationary limit coincides with the resonant ordering in Ref. 17 for the zonal flow amplitude It is used in Ref. 17 for estimating the nonlinear upshift.

Our notations are: $\rho_{s}=c_{s} / \Omega_{c i}$, ion sound speed $c_{s}$ $=\sqrt{T_{e} / m_{i}}$, ion gyro-frequency $\Omega_{c i}=e B / m_{i}, \mathrm{v}_{\mathrm{di}}$ and $\mathrm{v}_{\mathrm{de}}$ are the ion and electron magnetic drift velocity, respectively. $\eta_{\mathrm{i}}$ is the ratio of the density gradient scale length and the temperature gradient scale length, i.e., $\eta_{i} \equiv n_{i}\left|\nabla T_{i}\right| /\left(T_{i}\left|\nabla n_{i}\right|\right)$. Note that the perturbed electric potential $\varphi$ has been normalized, i.e., $\phi=\mathrm{e} \varphi / T_{e}$. Here, we assumed Boltzmann electrons, thus keeping only the Hasegawa-Mima nonlinearity.

We will here be mainly concerned with flows. The lowest orders will here give the linear dispersion relation and the group velocity $\lambda$.

To first order we get the linear dispersion relation (see Ref. 6 page 160 for the derivation)

$$
\begin{aligned}
\omega^{2}+ & \frac{10}{3 \tau} \omega \omega_{d}+\frac{5}{3 \tau^{2}} \omega_{d}^{2}-\frac{5}{3 \tau} k_{\|}^{2} c_{s}^{2}\left(1+\frac{\omega_{d}}{\tau \omega}\right) \\
= & \omega\left(\omega_{* n}-\omega_{d e}\right)-\frac{1}{\tau}\left(\eta_{i}-\frac{7}{3}+\frac{5}{3} \varepsilon_{n}\right) \omega_{* n} \omega_{d} \\
& -k_{\perp}^{2} \rho_{s}^{2}\left(\omega+\frac{1+\eta_{i}}{\tau} \omega_{* n}\right)\left(\omega+\frac{5}{3 \tau} \omega_{d}\right) \\
+ & {\left[\omega+\frac{5}{3 \tau} \omega_{d}+\frac{1}{\tau}\left(\eta_{i}-\frac{2}{3}\right) \omega_{* n}\right] \frac{k_{\|}^{2} c_{s}^{2}}{\omega} . }
\end{aligned}
$$


Here we introduced $\omega_{\mathrm{xn}}$ as the electron diamagnetic drift frequency with only density gradient and $\tau=\mathrm{T}_{\mathrm{e}} / \mathrm{T}_{\mathrm{i}}$. In order to obtain an eigenvalue equation ${ }^{41}$ we now temporarily introduce polar coordinates:

$$
y \rightarrow r \theta ; k_{\|} \rightarrow-\frac{i}{q R} \frac{\partial}{\partial \theta}
$$

After solving the quadratic eigenvalue solution in strong ballooning we have for the unstable branch ${ }^{6}$

$$
\begin{gathered}
\omega=\omega_{L}=\frac{\omega_{* n}}{2}\left[1-\varepsilon_{n}\left(1+\frac{10}{3 \tau}\right)\right. \\
\left.-\frac{1}{\tau}\left(1+\eta_{i}+\frac{5}{3} \varepsilon_{n}\right) k_{y}^{2} \rho_{s}^{2}\right]-i \gamma_{d}, \\
\gamma_{d}=\left(1+\frac{5}{3 \tau}\right) \frac{\varepsilon_{n}|s|}{4 q} \omega_{* n},
\end{gathered}
$$

where $\mathrm{q}$ is the safety factor and $\mathrm{s}=(\mathrm{r} / \mathrm{q}) \mathrm{dq} / \mathrm{dr}$ where $\mathrm{r}$ is the local plasma radius.

By differentiating (16) we get the group velocity

$$
\begin{aligned}
\lambda= & \frac{\partial \omega}{\partial k_{y}}=\frac{\omega}{k_{y}}-\frac{1}{k_{y}}\left[\frac{k_{y}^{2} \rho_{s}^{2}}{\omega\left(1+k_{y}^{2} \rho_{s}^{2}\right)-\omega_{L}}\left(\omega+\frac{5}{3 \tau} \omega_{d e}\right)\right. \\
& \left.\times\left(\omega+\frac{1+\eta_{i}}{\tau} \omega_{* n}\right)\right] .
\end{aligned}
$$

We finally obtain our equation for the flow dynamics from the vorticity equation to order $\varepsilon^{2}$.

We introduce

$$
\begin{aligned}
U= & \left(\mathrm{v}_{\mathrm{n}}-\mathrm{v}_{\mathrm{de}}\right)\left[\lambda\left(1+\frac{5}{3 \tau}\right)-\frac{5}{3} \mathrm{v}_{\mathrm{di}}\left(1+\frac{1}{\tau}\right)\right] \\
& +\left(\eta_{i}-\frac{2}{3}\right) \mathrm{v}_{\mathrm{n}}\left(\mathrm{v}_{\mathrm{di}}+\frac{\lambda}{\tau}\right),
\end{aligned}
$$

where $\mathrm{v}_{\mathrm{n}}$ is the electron diamagnetic drift with only density gradient. Integration over $\xi$ and using the linear relation (2d) finally leads to the excitation level of zonal flows.

We assume a standing wave structure in the $\mathrm{x}$ direction with box size $\mathrm{L}$

$$
\widehat{\varphi}_{1}^{1}(x, \xi, \tau)=\phi(\xi, \tau) \sqrt{\frac{2}{L}} \sin \left(k_{m} x\right) .
$$

We find that the stationary state, where the $\zeta$ derivative vanishes, is the same as the nonresonant state, i.e.,

$$
\begin{gathered}
\hat{\varphi}_{0}^{(2)}=F \frac{\partial}{\partial x}\left|\widehat{\phi}_{1}^{(1)}\right|^{2} \\
F=\frac{k_{y}{ }^{2} \rho_{s}^{2} c_{s}[1+\Gamma(\omega)] k_{m} L}{k_{m}\left[\lambda+\frac{1}{\tau}\left(1+\eta_{i}\right) \mathrm{v}_{\mathrm{n}}-\frac{U}{4 k_{m}^{2} \rho_{s}^{2} N} \mathrm{v}_{\mathrm{di}}\right]} \sin \left(2 k_{m} x\right),
\end{gathered}
$$

where $\Gamma(\omega)=\frac{1}{\omega-\frac{5}{3} \omega_{D i}}\left[\frac{2}{3} \omega+\left(\eta_{i}-\frac{2}{3}\right) \omega_{* e}\right]$ and $N=\lambda\left[\lambda-\frac{10}{3} \mathrm{v}_{\mathrm{di}}\right]$ $+\frac{5}{3} \mathrm{v}_{\mathrm{di}}^{2}$.

We here took the second harmonic part in the $\mathrm{x}$ variation since that gives stronger flow shear. This is the nonresonant solution for the excitation of zonal flows by ITG modes. We here still have the resonance from the fluid resonance in the energy equation as contained in $\Gamma$. This resonance here comes from the diamagnetic drift in the Reynolds stress. Although the corresponding resonance in the energy equation is quadratic, the resonance from the Reynolds stress is wider and has turned out to be more important in numerical simulations.

For practical purposes we, however, use Eq. (8), which has a more general ordering and has been widely tested, for this purpose.

\section{NUMERICAL RESULT}

Our main result for the excitation of zonal flows is Eq. (22). Thus we take the radial electric field, $\mathrm{E}_{\mathrm{r}}$ as

$$
E_{r}=-\frac{\partial \varphi_{0}{ }^{(2)}}{\partial r}
$$

and use (13) to get the flow shear. As it turns out, the present ordering is not completely identical to the ordering in the numerical solution. Thus, while the numerical solution used $\mathrm{k}_{\theta} \rho_{\mathrm{s}}=0.3$ (actually $\left.(0.1)^{0.5}=0.316\right)$ and $\mathrm{k}_{\mathrm{m}}=\mathrm{k}_{\theta}$, we have here used $\mathrm{k}_{\mathrm{m}}=\mathrm{k}_{\theta} / 2^{0.5}$ for the analytical solution. This may sometimes be realistic near marginal stability before the nonlinear mixing has reached full strength. Then, using the Cyclone base-case data we obtain the result in Fig. 1.

\section{A. Gyro fluid resonance}

As is seen from Fig. 1 there is a very strong resonance at marginal linear stability in the reactive model. We are now

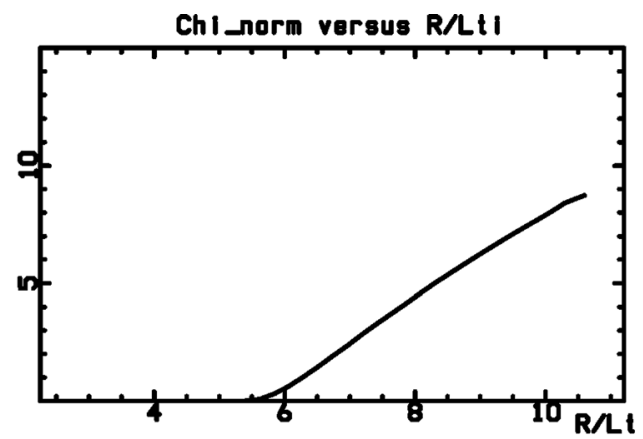

CHIYC

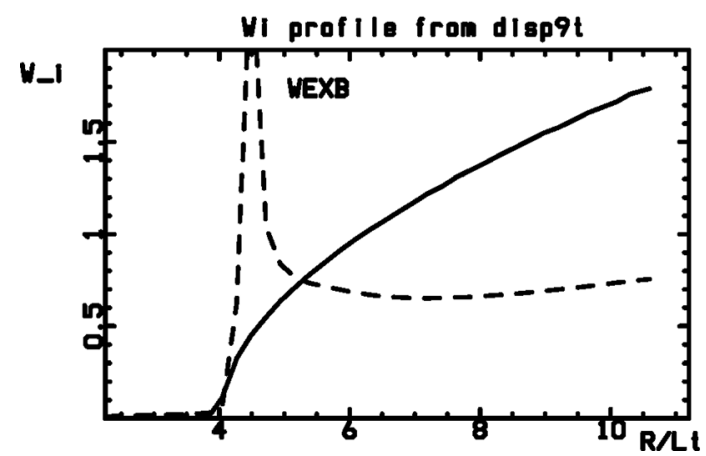

FIG. 1. (a) Ion thermal conductivity for a scan in the Cyclone (Ref. 10) gradient. (b) Linear growth rate (full line) and flow shear (dashed line) for the same scan as in (a). 
interested in comparing with a gyro-Landau fluid model. The main reason for this is to show that zonal flows are much weaker in gyro-Landau fluid models in order to explain the vast difference in normalization which had to be introduced artificially for gyro-Landau fluid models. Here we use the gyro fluid resonance according to Refs. 33 and 34. We have previously used these in Ref. 11 . The model is

$$
\nabla \cdot \mathbf{q}_{j}=\nabla \cdot \mathbf{q}_{* j}+\nabla \cdot \mathbf{q}_{G f}
$$

where

$$
\nabla \cdot \mathbf{q}_{G f}=\frac{9}{8}(-\sqrt{2}+i) \omega_{D e} n \delta T_{i}
$$

After adding the gyro-Landau fluid resonance (24) the resonance in Fig. 1 changes significantly as shown by Fig. 2.

Our first observation is here that while the reactive nonlinear upshift, in Fig. 1(a), is close to that of Dimits (threshold near 6), the nonlinear upshift in Fig. 2(a) is close to that of the IFS-PPPL model in Ref. 12. However, the most remarkable difference here is the difference between the flow shear (wexb) in Figs. 1(a) and 2(a). The reason is that here the dissipative resonance enters in the denominator of (2d) thus detuning the resonance in the temperature perturbation which enters in the Reynolds stress (8). As was pointed out in Ref. 17, our fluid model amounts to treating the fluid resonance as exact, thus allowing us to go infinitely close to it. Thus, we expect the gyro-Landau resonance to detune it strongly. However, a consequence of treating the reactive fluid resonance as exact is that we must include it by multiplying by the denominator. This, of course, implies that also the numerator of $(2 \mathrm{~d})$ will have to vanish at the pole. The

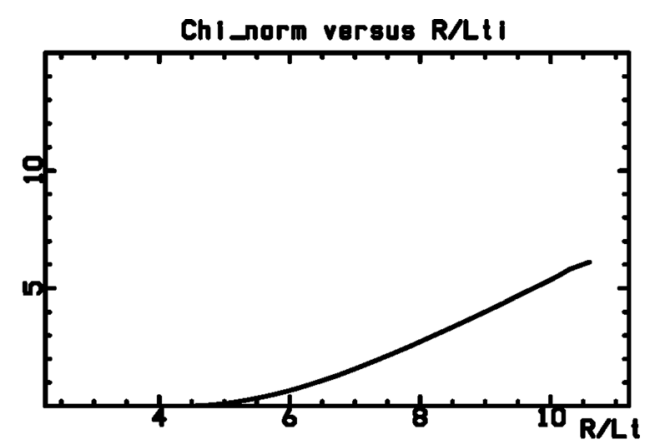

CHIYC

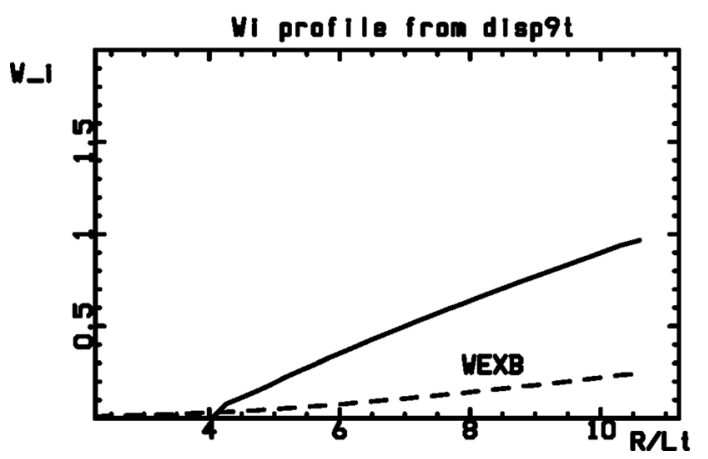

FIG. 2. (a) Same scan as in Fig. 1(a) but including the gyro-Landau resonance (24). (b) Same scan as in Fig. 1(b) but now again using (24). present calculation verifies that we still have a strong singularity at the fluid resonance. The same result was, in fact, obtained in Ref. 13 but there we calculated a zonal flow from the convective nonlinearity in the energy equation. However, the resonance was the same, i.e., the pole in $(2 \mathrm{~d})$. Thus, although the nonlinear upshifts in the reactive and gyroLandau fluid models were in good agreement with Ref. 12 it is actually the very strong difference at marginal stability that is of main interest here. This is since the zonal flow in the reactive model is so much stronger than that in the gyro-Landau fluid model This is the reason for the need to normalize gyro-Landau fluid models against nonlinear gyrokinetics. ${ }^{29,30}$ We have already noticed that Landau damping gives an increased transport in the Hasegawa-Wakatani model ${ }^{11}$ since it introduces an asymmetry between waves propagating in the two possible poloidal directions. In our case we get a very strong difference in the strength of zonal flows when dissipative wave-particle resonances are introduced. The validity of our reactive closure was verified very clearly in Ref. 21. Another point is that the gyro-Landau fluid model here gives much less overall transport than it gave in the Cyclone tests. This is because the gyro-Landau fluid model here was normalized in the same way as the reactive model in Fig. 1 while, in the Cyclone work, it had its natural normalization where the weak zonal flows were not able to create an absorbing boundary for long wavelengths.

\section{CONCLUSIONS}

Recent simulations of internal transport barriers on JET, the L-H transition on EAST, and the Dimits nonlinear upshift in the Cyclone work have, as pointed out previously, shown the importance of including the temperature perturbation in the Reynolds stress. In fact, the fluid closure, as expressed by the reactive resonance in (1) is of utmost importance for dynamic phenomena of drift waves involving temperature dynamics in magnetized plasmas. The most easily interpreted phenomena is the Dimits nonlinear upshift. The first attempt to understand the upper boundary of this was the effect of higher order nonlinear decay of the zonal flow, ${ }^{31}$ ignoring drift terms that are of the same order as the basic generating terms. A more recent attempt was made in Ref. 33 where the formulation goes directly into drift wave action. Also here further nonlinear effects were introduced in terms of a renormalized frequency broadening. However, these authors also included the temperature dependence of the Reynolds stress and it will be interesting to see if a modified isotope effect will eventually come out of this. We here recall that the nonlinear upshift in the Cyclone simulations was very sensitive to the resolution in terms of the number of particles in the codes. The upshift area was more sensitive to this resolution than other regions in the graph. This clearly indicates that the nonlinear upshift is closely related to the wave particle resonance in the kinetic simulations. This is directly transferred to the reactive fluid resonance in our simulations as a consequence of our derivation of the fluid closure in Ref. 21.

Thus, as far as we can see, our simulation of the Dimits shift in Ref. 14 was the first including the temperature 
dynamics consistently. We have here shown that we can derive a strong excitation of the background flow potential $\varphi$ by systematic and often used ordering relations giving good agreement with both Dimits original result and our own previous simulation results. The main conclusion that the limitation of the Dimits shift is due to the detuning of the fluid resonance is confirmed. The fluid resonance in the energy equation enters through $\Gamma$ giving enhanced excitation close to the resonance. The procedure of the calculations is to calculate the radial electric field in (12) and from there the flow shear from Eq. (13). We have then used the Waltz rule ${ }^{37-39}$ to obtain the stabilization of the drift waves caused by the flow shear (13). The resonance of the potential $\varphi$ at the fluid resonance is the same as obtained in transport simulations giving the $\mathrm{L}-\mathrm{H}$ transition on EAST ${ }^{35}$ and the nonlinear Dimits upshift. ${ }^{12}$ It is important that we have here taken the nonlinear temperature dynamics from the Reynolds stress as the convected diamagnetic flow. This has turned out to be the most relevant effect as found in transport simulations. ${ }^{14,35,36}$

Thus, is summary, the fluid resonance in the energy equation is instrumental in obtaining internal and edge (H-mode) transport barriers and also the Dimits nonlinear upshift. As found in the present work, the zonal flows get so much stronger in our reactive model than in gyro-Landau fluid models that they must be the reason for the absorbing boundary condition for long wavelengths in the reactive model. This explains why gyro-Landau fluid models have to be normalized against nonlinear kinetic theory as shown in Refs. 29 and 30.

The good properties of the model, already in its original $2 \mathrm{~d}$ version, are due to the fact that parallel ion motion is subdominant and enters only near marginal stability. For internal barriers, this means that small magnetic shear can trigger a barrier since it is stabilizing. However, for edge barriers and the Dimits shift, the flow shear comes first and takes the modes near marginal stability. Thus, here parallel ion motion is not needed for obtaining the phenomenon as such but only for precision. This means that already our first simple model in Ref. 4 would be able to describe the Dimits shift at least qualitatively if we add the dynamics of poloidal rotation. Thus, the original model in Ref. 4 still has an appreciable regime of validity!

\section{ACKNOWLEDGMENTS}

The authors are grateful for discussions with Professor C.S. Liu.

\footnotetext{
${ }^{1}$ A. Hasegawa and K. Mima, "Pseudo-three-dimensional turbulence in magnetized inhomogeneous plasma," Phys. Fluids 21, 87 (1978).

${ }^{2}$ A. Hasegawa, C. G. Maclennan, and Y. Kodama, "Non-linear behaviour and turbulence spectra of drift waves and Rossby waves," Phys. Fluids 22, 2122 (1979).

${ }^{3}$ P. H. Diamond, S. I. Itoh, K. Itoh, and T. S. Hahm, "Zonal flows in plasma-A review," Plasma Phys. Controlled Fusion 47, R35 (2005).

${ }^{4}$ J. Weiland and H. Nordman, "Transport due to fully toroidal drift waves," in Proceedings of the Varenn Lausanne Workshop on Theory in Fusion Plasmas, Chexbres, Switzerland (1988), p. 451.

${ }^{5}$ A. Zagorodny and J. Weiland, "Statistical theory of turbulent transport (non-Markovian effects)," Phys. Plasmas 6, 2359 (1999).

${ }^{6} \mathrm{~J}$. Weiland, Stability and Transport in Magnetic Confinement Systems (Springer, New York, 2012).
}

${ }^{7}$ J. Weiland, A. Zagorodny, V. Zasenko, and N. Shatashvili, "On the Physics description of fusion plasmas," AIP Conf. Proc. 1445, 34 (2012).

${ }^{8} \mathrm{~J}$. Weiland, "Turbulent transport in fusion plasmas, effects of toroidicity and fluid closure," AIP Conf. Proc. 1188, 193 (2009).

${ }^{9}$ A. Hasegawa and M. Wakatani, Phys. Rev. Lett. 50, 682 (1983).

${ }^{10} \mathrm{~J}$. W. Connor and O. P. Pogutse, "On the relationship between mixinglength and strong turbulence estimates for transport due to drift turbulence," Plasma Phys. Controlled Fusion 43, 155 (2001).

${ }^{11}$ M. Wakatani and A. Hasegawa, "A collisional drift wave description of plasma edge turbulence," Phys. Fluids 27, 611 (1984).

${ }^{12}$ A. M. Dimits, G. Bateman, M. A. Beer, B. I. Cohen, W. Dorland, G. W. Hammett, C. Kim, J. E. Kinsey, M. Kotschenreuther, A. H. Kritz, L. Lao, J. Mandrekas, W. M. Nevins, S. E. Parker, A. J. Redd, D. E. Shumaker, R. Sydora, and J. Weiland, "Comparisons and physics basis of tokamak transport models and turbulence simulations," Phys. Plasmas 7, 969 (2000).

${ }^{13}$ R. Moestam, D. Shaik, and J. Weiland, "Self consistent theory of zonal flows in ion temperature gradient turbulence," Phys. Plasmas 11, 4801 (2004).

${ }^{14}$ G. Q. Wang, J. Ma, and J. Weiland, "Zonal flows near marginal stability in drift wave transport," Phys. Scr. 90, 065604 (2015).

${ }^{15} \mathrm{G}$. Q. Wang, Ma. Jun, J. Weiland, and A. Zagorodny, "Excitation of zonal flows by ion-temperature gradient modes excited by the fluid resonance," Chin. Phys. Lett. 32, 115201 (2015).

${ }^{16} \mathrm{~J}$. P. Mondt and J. Weiland, "Two fluid theory of thermal transport in current-carrying edge plasma," Phys. Scr. 39, 92 (1989).

${ }^{17} \mathrm{~J}$. Weiland, "Thermal instabilities in drift wave turbulence," Phys. Scr. 90, 088009 (2015).

${ }^{18} \mathrm{~J}$. Weiland, "Review of mixing length estimates and effects of toroidicity in a fluid model for turbulent transport in tokamaks," Plasma Phys. Rep. 42, 502 (2016).

${ }^{19}$ N. Mattor and S. Parker, Phys. Rev. Lett. 79, 3419 (1997).

${ }^{20}$ I. Holod, J. Weiland, and A. Zagorodny, "Nonlinear Fluid Closure: Threemode slab ion temperature gradient problem with diffusion," Phys. Plasmas 9, 1217 (2002).

${ }^{21}$ J. Weiland, C. S. Liu, and A. Zagorodny, "Transition from a coherent three wave system to turbulence with application to the fluid closure," J. Plasma Phys. 81, 905810101 (2015).

${ }^{22}$ J. Weiland, A. Jarmen, and H. Nordman, "Diffusive particle and heat pinch effects in toroidal plasmas," Nucl. Fusion 29, 1810 (1989).

${ }^{23}$ G. Tardini, A. G. Peeters, G. V. Pereverzev, F. Ryter, and the ASDEX Upgrade Team, "Theory based modelling of ASDEX Upgrade discharges with ECH modulation," Nucl. Fusion 42, L11 (2002).

${ }^{24} \mathrm{~J}$. Weiland and H. Nordman, "Drift wave model for inward energy transport in tokamak plasmas," Phys. Fluids B5, 1669 (1993).

${ }^{25} \mathrm{~J}$. Weiland, A. Zagorodny, and V. Zasenko, "Fluid and kinetic modelling on timescales longer than the confinement time in bounded systems," AIP Conf. Proc. 1177, 96 (2009).

${ }^{26} \mathrm{~J}$. Weiland, P. Mantica, and the JET-EFDA Contributors, "Effects of flow shear on the correlation length of drift wave turbulence," in 38th EPS Conference, Strassbourg, France, 27 June-1 July 2011, paper No. P5.130.

${ }^{27}$ G. M. Staebler, R. E. Waltz, and J. E. Kinsey, "New paradigm for suppression of gyrokinetic turbulence by velocity shear," Phys. Rev. Lett. 110, 055003 (2013).

${ }^{28}$ K. Nozaki and T. Taniuti, "Plasma flow and a soliton in a theta pinch," J. Phys. Soc. Jpn. 46, 970 (1979).

${ }^{29}$ J. Kinsey, G. M. Staebler, and R. E. Waltz, "Predicting core and edge transport barriers in tokamaks using the glf 23 drift wave transport model," Phys. Plasmas 12, 052503 (2005).

${ }^{30}$ ITER Physics Basis Editors, "Progress in the ITER physics basis, II confinement," Nucl. Fusion 47, S1-S413 (2007).

${ }^{31}$ B. N. Rogers, J. F. Drake, and A. Zeiler, "Phase space of tokamak edge turbulence, the L-H transition and the formation of the edge pedestal," Phys. Rev. Lett. 81, 4396 (1998).

${ }^{32}$ B. N. Rogers, W. Dorland, and M. Kotschenreuther, "Generation and stability of zonal flows in ion-temperature-gradient mode turbulence," Phys. Rev. Lett. 85, 5336 (2000).

${ }^{33}$ S.-I. Itoh and K. Itoh, "Hydrogen isotope effect on the Dimits shift," Nucl. Fusion 56, 106028 (2016).

${ }^{34}$ F. Wagner, G. Becker, K. Behringer, D. Campbell, A. Eberhagen, W. Engelhardt, G. Fussmann, O. Gehre, J. Gernhardt, G. V. Gierke, G. Haas, M. Huang, F. Karger, M. Keilhacker, Q. Kluber, M. Kornherr, K. Lackner, G. Lisitano, G. G. Lister, H. M. Mayer, D. Meisel, E. R. Müller, H. Murmann, H. Niedermeyer, W. Poschenrieder, H. Rapp, H. Röhr, F. Schneider, G. Siller, E. Speth, A. Stäbler, K. H. Steuer, G. Venus, O. 
Vollmer, and Z. Yü, "Regime of improved confinement and high beta in neutral beam-heated divertor discharges of the ASDEX Tokamak," Phys. Rev. Lett. 49, 1408 (1982).

${ }^{35}$ J. Weiland, K. Crombe, P. Mantica, V. Naulin, T. Tala, and the JETEFDA Contributors, "Comparison of edge and internal transport barriers in drift wave predictive simulations," AIP Conf. Proc. 1392, 85 (2011).

${ }^{36} \mathrm{~J}$. Weiland, "Simulations of the L-H transition on experimental advanced superconducting Tokamak," Phys. Plasmas 21, 122501 (2014).

${ }^{37}$ T. Rafiq, A. H. Kritz, J. Weiland, A. Y. Pankin, and L. Luo, "Physics basis of Multi-Mode anomalous transport module," Phys. Plasmas 20, 032506 (2013).
${ }^{38}$ R. E. Waltz, R. R. Dominguez, and G. W. Hammett, "GyroLandau fluid models for toroidal geometry," Phys. Fluids B4, 3138 (1992).

${ }^{39}$ R. E. Waltz, G. D. Kerbel, and J. Milovich, "Toroidal gyro-Landau fluid model turbulence simulations in a nonlinear ballooning mode representation with radial modes," Phys. Plasmas 1, 2229 (1994).

${ }^{40} \mathrm{~A}$. Zagorodny and J. Weiland, "Non-Markovian renormalization of kinetic coefficients for drift-type turbulence in magnetized plasmas," Phys. Plasmas 16, 052308 (2009).

${ }^{41}$ J. Weiland, "Analytical eigenvalue solution for ITG modes of general modewidth," Phys. Plasmas 11, 3238 (2004). 